\title{
Influence of pharmacological manipulations of NMDA and cholinergic receptors on working versus reference memory in a dual component odor span task
}

\author{
David A. MacQueen, ${ }^{1,3}$ Savannah R. Dalrymple, ${ }^{2,3}$ David J. Drobes, ${ }^{1,3,4}$ and David \\ M. Diamond ${ }^{2,3,5,6}$ \\ ${ }^{1}$ Moffitt Cancer Center, Tampa, Florida 33612, USA; ${ }^{2}$ Medical Research Service, VA Hospital, Tampa, Florida 33612, USA; \\ ${ }^{3}$ Department of Psychology; ${ }^{4}$ Department of Oncologic Sciences; ${ }^{5}$ Department of Molecular Pharmacology and Physiology; ${ }^{6}$ Center for \\ Preclinical and Clinical Research on PTSD, University of South Florida, Tampa, Florida 33620, USA
}

\begin{abstract}
Developed as a tool to assess working memory capacity in rodents, the odor span task (OST) has significant potential to advance drug discovery in animal models of psychiatric disorders. Prior investigations indicate OST performance is impaired by systemic administration of $\mathrm{N}$-methyl-D-aspartate receptor (NMDA-r) antagonists and is sensitive to cholinergic manipulations. The present study sought to determine whether an impairment in OST performance can be produced by systemic administration of the competitive NMDA-r antagonist 3-(2-carboxypiperazin-4-yl)propyl-1-phosphonic acid (CPP; 3, 10, $17 \mathrm{mg} / \mathrm{kg}$ i.p.) in a unique dual-component variant of the OST, and whether this impairment is ameliorated by nicotine $(0.75 \mathrm{mg} / \mathrm{kg}$ i.p.). Male Sprague-Dawley rats were trained to asymptotic level of performance on a 24-trial two-comparison incrementing nonmatching to sample OST. In addition, rats were administered a two-comparison olfactory reference memory (RM) task, which was integrated into the OST. The RM task provided an assessment of the effects of drug administration on global behavioral measures, long-term memory and motivation. Several measures of working memory (span, longest run, and accuracy) were dose dependently impaired by CPP without adversely affecting RM. Analysis of drug effects across trial blocks demonstrated a significant impairment of performance even at low memory loads, suggesting a CPP-induced deficit of olfactory short-term memory that is not load-dependent. Although nicotine did not ameliorate CPP-induced impairments in span or accuracy, it did block the impairment in longest run produced by the $10 \mathrm{mg} / \mathrm{kg}$ dose of CPP. Overall, our results indicate that performance in our 24 odor two-comparison OST is capacity dependent and that CPP impaired OST working, but not reference, memory.
\end{abstract}

The odor span task, which was originally developed by Dudchenko et al. (2000), makes use of a primary sensory modality for rodents (olfaction) to assess the neurobiological and neuropharmacological basis of rodent memory. In the original procedure, rats were trained to dig in cups of sand, each mixed with a different household spice (e.g., basil or paprika), to retrieve buried food rewards. On each successive trial, a novel odor was presented, along with every odor that had been presented in preceding trials, but only responses to the novel odor were reinforced. To respond accurately on any given trial the rat needed to remember each stimulus it had encountered earlier in the session and withholds responses to these stimuli. Therefore, memory demands escalated over the course of the procedure as the number of odors the rat was required to remember increased. Dudchenko et al. (2000) demonstrated that performance in the task declined across trials, indicating that task accuracy was negatively impacted by increasing memory load. However, in this OST procedure, the number of comparisons presented on each successive trial increases in tandem with the number of stimuli to remember, confounding the relationship between accuracy and memory load. By limiting the number of comparisons presented on each trial, chance performance can be equated across trials of the task. Using this proce-

Corresponding author: ddiamond@mail.usf.edu Article is online at http://www.learnmem.org/cgi/doi/10.1101/Im.041251.115. dure, the negative association of performance and memory load has been confirmed (MacQueen et al. 2011).

Although the specific brain regions involved in this task have yet to be fully identified, there is evidence for the involvement of the prefrontal cortex and the hippocampus in the OST and its variations. Studies have shown that disruption of the medial prefrontal cortex (mPFC) by either chemical inactivation or administration of GluN2B antagonists impairs span without disrupting latency to choice or odor sensitivity in nonmatch-to-sample OST variations (Davies et al. 2013a,b). Although the effects of mPFC manipulation on OST performance are unambiguous, the contribution of the hippocampus, a structure well established in the processing of spatial memories (Martin and Clark 2007), is less clear. In an assessment of nonspatial working memory, Olton and Feustle (1981) reported impaired accuracy in a radial arm maze when fimbria-fornix lesioned rats were required to utilize cue strategies to remember previous arm entries. In contrast, performance in the nonmatch to sample OST developed by Dudchenko et al. (2000) was not impaired by hippocampal

C 2016 MacQueen et al. This article is distributed exclusively by Cold Spring Harbor Laboratory Press for the first 12 months after the full-issue publication date (see http://learnmem.cshlp.org/site/misc/terms.xhtml). After 12 months, it is available under a Creative Commons License (AttributionNonCommercial 4.0 International), as described at http://creativecommons. org/licenses/by-nc/4.0/. 
lesions. Moreover, animals with lesions to the ventral hippocampus exhibited impairments in accuracy with a match to sample variant in which responses to the odor presented earliest in the sequence were reinforced (Fortin et al. 2002; Kesner et al. 2010, 2011). Kesner et al. (2010) also report impaired accuracy in ventral CA1 lesioned rats for odors presented in close temporal proximity (see also Kesner et al. 2002). Thus, the involvement of hippocampal and frontal regions in the OST appears to depend on the requirements of the task itself: variants in which rodents must respond to a novel odor appear to require functioning of the PFC, whereas tasks requiring the rodent to remember odors for longer periods of time or to remember the temporal sequence of odors involve subregions of the hippocampus, as well.

Pharmacological studies in rodents have indicated that NMDA receptor activation is required for intact performance in the OST. MacQueen et al. (2011) examined the effects of the noncompetitive $N$-methyl-D-aspartate receptor (NMDA-r) antagonist MK-801 on an OST in rats. Their procedure included a control for sensorimotor and motivational drug effects in the form of well-learned two-comparison olfactory reference memory task [RMT; referred to by MacQueen et al. (2011) as simple discrimination] inserted intermittently throughout a 24 trial (fixed-trial) OST procedure. Accurate responding in simple discrimination trials demonstrated intact motivation for reward, as well as sensorimotor competence and intact long-term memory. MK-801 produced a dose-dependent impairment of accuracy and span in the OST, but did not disrupt simple discrimination performance, indicating that working memory was selectively impaired. Additional studies have confirmed selective effects of noncompetitive NMDA-r antagonists, such as MK-801 (dizocilpine) and ketamine, on working memory, while global (nonselective) impairments of OST performance have been reported for drugs with non-NMDA-r actions, including morphine, chlordiazepoxide, scopolamine, or MDMA (Galizio et al. 2013; Hawkey et al. 2014). These studies demonstrate that working memory in the OST is selectively impaired by noncompetitive NMDA-r antagonists. However, one study characterizing the effects of MK-801 on performance across memory load failed to find an interaction between dose and memory load, suggesting that drug effects were not load-dependent (MacQueen et al. 2011).

The competitive NMDA-r antagonist 3-(2-carboxypiperazin4-yl)propyl-1-phosphonic acid (CPP), which has previously been shown to impair memory (Larkin et al. 2008; Niimi et al. 2008; Feldman et al. 2010; Velazquez-Zamora et al. 2011) and to block long-term potentiation (Brun et al. 2001), has also been shown to impair span in a study using a variable trial adaptation of the OST. Davies et al. (2013a) demonstrated that systemic administration of CPP or the GluN2B-selective antagonist Ro 25-6981 impaired span without affecting nonspecific performance measures, indicating that competitive NMDA-r antagonists produce effects selective to the OST, as well.

Performance in the OST has also been studied in relation to the overexpression of NMDA-r in mice. In an investigation of the contribution of NMDA-r subunits to long-term potentiation and memory, Cui et al. (2011) reported that mice with overexpression of the NMDA-r 2B subunit produced longer spans, greater accuracy and fewer errors on a 20-odor span task, as well as greater in vitro LTP in prefrontal cortex when compared with control animals. With this and other studies of NMDA-r antagonism, OST performance appears to be sensitive to NMDA-r function, possibly due the primary role of this receptor in synaptic plasticity within the prefrontal cortex and hippocampus.

Odor span is also susceptible to cholinergic manipulations. Selective knockout of the $\alpha 7$ nicotinic acetylcholine receptor (nAChR) subunit gene in mice impaired acquisition and performance of a 12-stimuli odor span task relative to wild-type controls
(Young et al. 2007a). In rats, nicotine and selective nAChR agonists improved span and reversed odor span deficits produced by administration of the NMDA-r antagonist, ketamine (Rushforth et al. 2011). These results indicate that manipulation of the nicotinic acetylcholine receptor can influence OST performance in rodents.

The present study had three goals: First, we sought to develop a two-comparison variant of the OST to assess the effects of pharmacological manipulations on working and reference memory, as well as sensorimotor and motivational processes. A fixed-trial variant of the OST in which only two-comparison stimuli, one familiar and one novel, were simultaneously presented allowed for characterization of performance across memory load. By presenting only two odors in each trial, performance across memory load remained uncorrupted by variations in chance performance inherent to the original OST and its previous adaptations. Additionally, our variant of the OST reduced session length and experimenter demands of task preparation without sacrificing the variety of measures that this task produces to assess memory. Second, we assessed the effects of systemic administration of CPP on working and reference OST memory. We tested the hypothesis that CPP would selectively impair working, but not reference, memory in the OST. Based upon observations of the effects of the noncompetitive antagonist MK-801, we hypothesized that performance would be selectively impaired at low memory loads with no evidence of load-dependent dose effects. Finally, we hypothesized that CPP-induced impairments in performance would be ameliorated by acute nicotine administration.

\section{Results}

\section{Training and baseline}

All eight rats progressed to asymptotic performance level on the dual-component 24 odor two-comparison OST (see Fig. 1). Training required an average of 25 sessions (range of 22-30

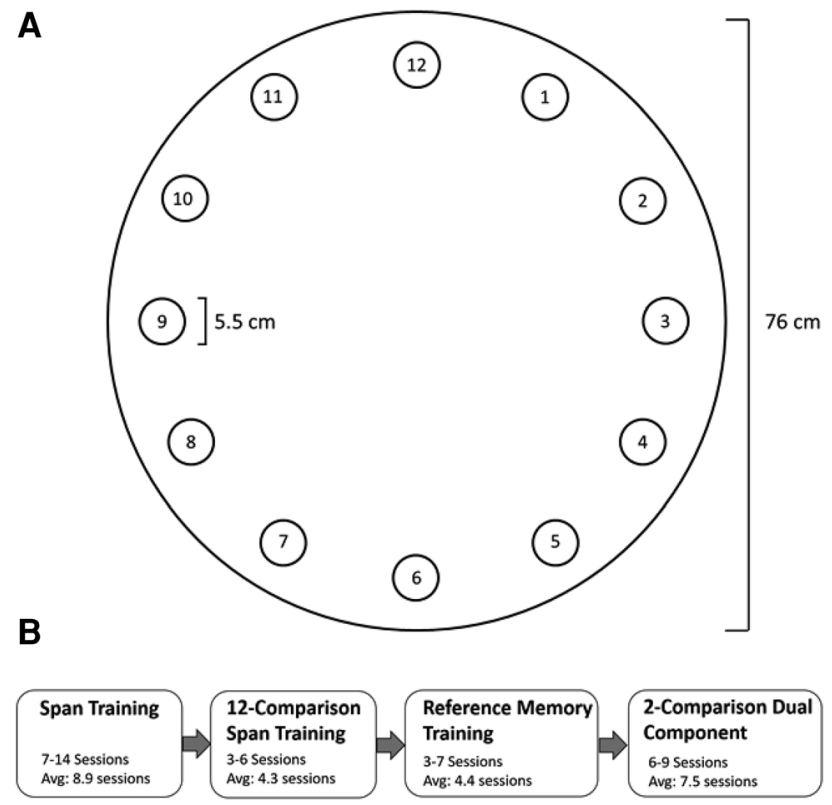

Figure 1. (A) Odor arena dimensions. (B) Timeline of OST training. The range and average number of sessions required to reach criterion for each training procedure are included. The number of sessions is equivalent to days required to reach criterion as one 30-min session was conducted each day, Monday through Friday (five sessions per week). 
sessions) and animals were tested for an average of 51.1 (range of 34 and 74) sessions of testing and drug manipulations. Performance remained consistent across periods of repeated testing and each rat continued to meet stability criteria once drug testing began. All baseline sessions were retained in analyses to produce the most reliable estimates of accuracy at each memory load. In terms of number of correct responses, rats produced an average span of $7.58( \pm 1.03)$, an average longest run of 10.89 ( \pm 0.94$)$, and an average percent correct performance of $83.2 \%$ $( \pm 1.92 \%)$ and $96.0 \%( \pm 0.75 \%)$ on the odor span and reference memory components, respectively, during baseline sessions. As depicted in Figure 2, there was a significant negative relationship between memory load and accuracy at baseline on the dualcomponent, two-comparison OST $\left(r^{2}=0.419, P<0.01\right)$.

\section{Drug effects}

Two-way repeated-measures ANOVAs were used to evaluate the effects of condition and component (odor span or reference memory) on percent correct accuracy, latency, and omissions. No significant effects of condition, component, or their interaction were observed for latency to choice or omissions (Ps $>0.05$; Fig. 3). A significant effect of condition $\left[F_{(6,42)}=5.90, P<0.001\right]$, component $\left[F_{(1,7)}=669.59, P<0.001\right]$, and their interaction $\left[F_{(6,42)}=7.08, P<0.001\right]$ was observed for percent correct accuracy (see Fig. 4). Separate one-way repeated-measures ANOVAs of odor span and reference memory performance revealed a significant effect of dose for odor span trials $\left[F_{(6,42)}=10.16, P<0.001\right]$ and no effect for reference memory trials. Average odor span accuracy after saline injections did not differ from accuracy on baseline sessions. Odor span accuracy at the 10 and $17 \mathrm{mg} / \mathrm{kg}$ doses of CPP was significantly impaired relative to both baseline and saline $(P s<0.05)$. Accuracy was also significantly impaired relative to both baseline and saline when nicotine was administered after the $17 \mathrm{mg} / \mathrm{kg}$ dose of CPP. When combined with nicotine, the $10 \mathrm{mg} / \mathrm{kg}$ dose of nicotine significantly impaired accuracy relative to baseline but, not saline. Accuracy at each of these doses after administration of nicotine did not significantly differ from accuracy at these doses without nicotine $(P>0.05)$.

Reference memory performance was not impaired at any of the CPP dose combinations tested. Therefore, the OST impairments observed in response to CPP administration cannot be accounted for by motivational, sensorimotor, or global cognitive effects of the drug and can therefore be considered to be selective to working memory. The dose-dependent effects of CPP on odor span performance were further characterized by comparing dose

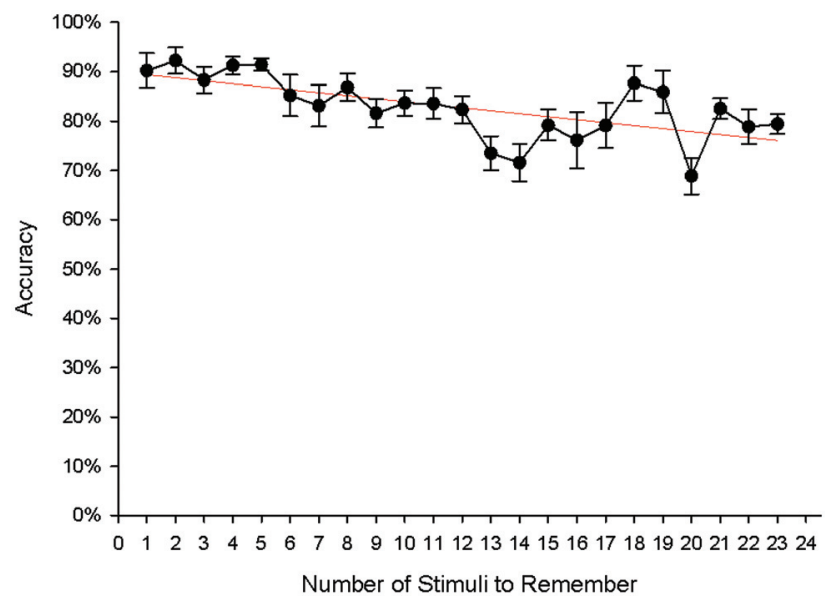

Figure 2. Mean \pm SEM accuracy by memory load and regression line. effects on accuracy across trial blocks (see Fig. 5). A two-way repeated-measures ANOVA revealed significant main effects for dose $\left[F_{(2,14)}=9.39, P<0.005\right]$ and trial block $\left[F_{(5,35)}=3.82\right.$, $P<0.01]$ but, no dose by trial block interaction $(P>0.05)$. As in earlier analyses, performance after the 10 and $17 \mathrm{mg} / \mathrm{kg}$ doses of CPP was significantly impaired relative to both baseline and saline $(P s<0.05)$. Performance during the first trial block was significantly better than performance during the third through final trial blocks and performance at the second trial block was better than at the fourth block ( $P \mathrm{~s}<0.05)$. First block performance was also compared across doses revealing a significant effect $\left[F_{(4,28)}=6.586, P<0.005\right]$ in which performance at the $17 \mathrm{mg} /$ $\mathrm{kg}$ dose was significantly impaired relative to both baseline and saline $(P s<0.05)$.

One-way repeated-measures ANOVAs were also used to examine the effects of condition on span, longest run, and stimulus cup approaches. A significant effect of condition was observed for both span $\left[F_{(3,18)}=5.761, P<0.010\right]$ and longest $\operatorname{run}\left[F_{(3,21)}=\right.$ $5.761, P<0.010]$. As illustrated in Figure 6, both span and longest run were significantly reduced relative to saline in response to the $17 \mathrm{mg} / \mathrm{kg}$ dose of CPP, with and without nicotine (Ps $<0.05)$. Longest run was also significantly impaired after the $10 \mathrm{mg} / \mathrm{kg}$ dose of CPP, which was blocked by administration of nicotine. A significant component by dose interaction was detected for approaches $\left[F_{(6,42)}=3.41, P<0.010\right]$. Separate one-way repeated-measures ANOVAs of approaches by component revealed a significant effect of dose on RM but, not WM trials. Post hoc analyses revealed that after administration of $17 \mathrm{mg} / \mathrm{kg} \mathrm{CPP}$, with or without nicotine, subjects sampled both RM odors before making a choice more frequently relative to baseline $(P s<0.05)$.

\section{Discussion}

We deployed a 24 odor two-comparison variant of the OST to assess the effects of pharmacological manipulations on working memory, to characterize performance across memory load, and to simultaneously provide an assessment of long-term memory, sensorimotor activity, and motivation. We found that administration of a competitive NMDA-r antagonist (CPP) produced dosedependent impairments in our variant of the OST. These findings are consistent with and extend prior odor span studies that have reported cognitive impairments in rats produced by competitive and noncompetitive NMDA-r antagonists (MacQueen et al. 2011; Rushforth et al. 2011; Davies et al. 2013a; Galizio et al. 2013) and enhanced performance in mice overexpressing the 2B NMDA-r subunit (Cui et al. 2011). Notably, CPP did not affect reference memory performance, demonstrating the selective involvement of NMDA receptors in the working memory component of the task.

Rats were trained on a 24 odor, two-comparison dualcomponent OST over an average of 25.0 sessions of training and 77.5 sessions of testing during the course of $\sim 6 \mathrm{mo}$. Chance performance was equated across the odor span and reference memory components of the task by limiting the number of comparisons presented on all trials. This adaptation of the OST procedure allowed for a comparison of performance across trials unbiased by concurrent variation in chance performance. Average baseline performance was similar to earlier studies using the dualcomponent procedure both in terms of span and percent correct accuracy (MacQueen et al. 2011; April et al. 2013; Galizio et al. 2013). Accuracy declined as the number of stimuli to remember increased, demonstrating that two-comparison odor span performance was capacity dependent. Thus, our two-comparison odor span procedure is sufficiently sensitive to the capacity effects reported from studies utilizing larger comparison arrays (MacQueen et al. 2011; Galizio et al. 2013). Notably, the decline 
Trial Latency and Omissions by Dose

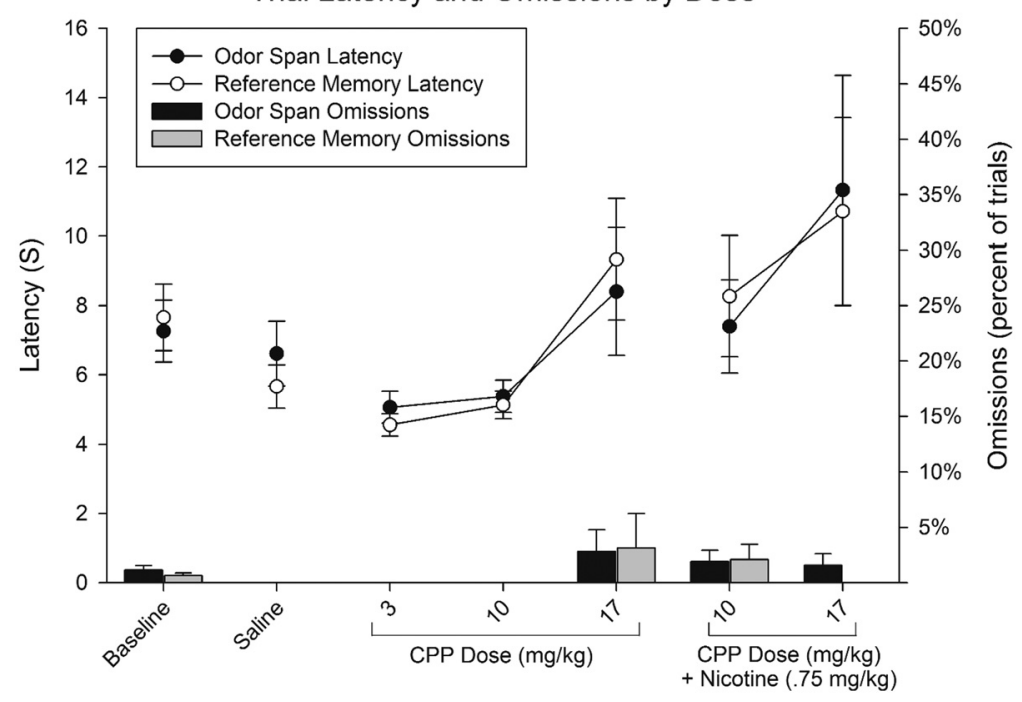

Figure 3. Latency to choice for completed odor span (filled circles) and reference memory (open circles) trials are depicted by dose (reference left axis). Percentages of trials in which the subject did not provide a response (omissions) are depicted for odor span (black bars) and reference memory (gray bars) trials (reference right axis).

in performance across memory load was modest and relatively linear. This contrasts with human performance of prototypical span tasks (e.g., digit span, spatial span, letter-number sequencing) in which accuracy declines precipitously after an initial error. Thus, the short-term memory processes utilized by the OST appear to be distinct from the limited capacity working memory stores hypothesized to underlie human performance on common visual, auditory, and spatial span tasks (Baddeley 2003).

The two-comparison OST design also allowed for a comparison of drug effects across trial blocks varying in memory load. While performance significantly declined with increasing memory load and dose of CPP, there was no interaction between these variables. Performance at the highest dose of CPP was impaired at the lowest memory loads, suggesting a profound deficit of olfactory short-term memory that was not load-dependent. The effects of CPP on performance across trial blocks were strikingly similar to the effects previously reported for the noncompetitive antagonist dizocilpine using a similar design (MacQueen et al. 2011). Thus, the present study indicates that with different mechanistic approaches toward NMDA-r blockade, systemic antagonist effects on the OST do not appear to be load-dependent.

It was possible that CPP effects on performance reflected a general impairment of attention produced by NMDA-r antagonism. However, the effect was restricted to short-term memory given that performance was spared in a concurrent reference memory task administered in a manner identical to OST trials. It is noteworthy that our two-comparison choice procedure facilitated a direct comparison of drug effects on all (WM and RM) trials since chance performance was maintained at 50\% for all WM and RM trials.

Our observations are consistent with prior reports of CPPinduced cognitive impairments (Larkin et al. 2008; Niimi et al. 2008; Feldman et al. 2010; Velazquez-Zamora et al. 2011) and LTP disruption in the hippocampus and prefrontal cortex (Brun et al. 2001). Additionally, Davies et al. (2013a) demonstrated that GluN2B receptors in medial prefrontal cortex were critical to OST performance. We speculate that the cognitive impairments reported here are due to the effect of CPP-induced NMDA-r antagonism on synaptic plasticity within the prefrontal cortex and potentially the hippocampus.
We also provided a preliminary assessment of the effects of nicotine on CPP-induced odor span deficits. Low doses of nicotine have been shown to enhance odor span performance (Rushforth et al. 2010) and to reverse NMDA-r antagonist-induced impairments (Rushforth et al. 2011) in rats. We observed evidence that a moderate dose of nicotine $(75 \mathrm{mg} / \mathrm{kg}$ i.p.) may reverse an impairment produced by CPP. Although span remained impaired when nicotine was administered after $\mathrm{CPP}$, the longest run measure was impaired at $10 \mathrm{mg} / \mathrm{kg}$ of $\mathrm{CPP}$, but not when this dose was coadministered with nicotine. Overall accuracy relative to baseline remained impaired during this combination, but the comparison with the saline-treated group was not statistically significant. In humans, nicotine enhancement of cognition is most pronounced in tasks of sustained attention and vigilance (Heishman et al. 2010). It is conceivable that recovery of the longest run measure without robust recovery of overall accuracy is related to the effects of nicotine on vigilance. However, nicotine did not ameliorate impairments on any measure at higher doses of CPP. Subsequent work is required to more fully examine the basis of interactions between CPP and nicotine.

Though the procedural features of the OST facilitated evaluation of the impact of memory load, it could be argued that training and testing of the two-comparison dual-component OST is overly time-consuming. However, it is worth noting that the overtraining of individual rats facilitated repeated testing and reduced confounds related to drug effects on task acquisition, as opposed to task demands. Using a within-subject training design allowed for repeated testing at the individual animal level, reducing the number of subjects needed to test manipulations. Moreover, the integrated dual-component feature of the task provided an assessment of both working and long-term (reference) memory within the same session, which reduced the need for testing of additional

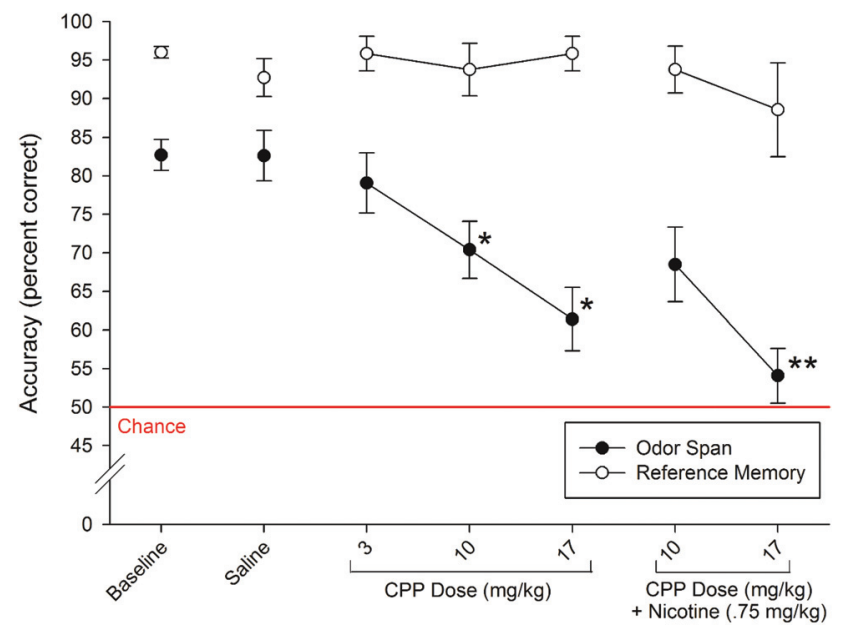

Figure 4. Percent correct accuracy \pm SEM across conditions for odor span and reference memory trials. Asterisks indicate a significant difference from saline $(*) P<0.05 ;\left(^{* *}\right) P<0.01$. 


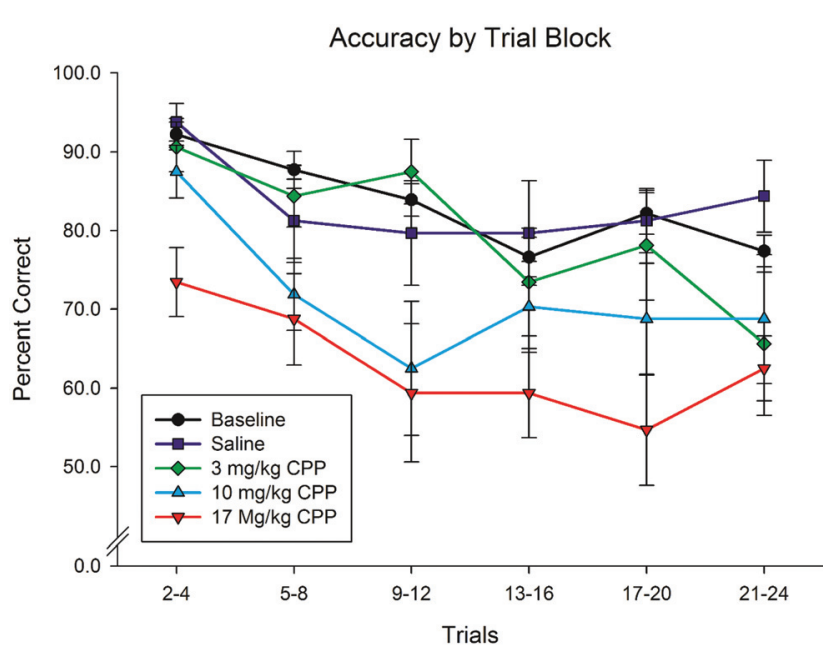

Figure 5. Average accuracy \pm SEM across trial blocks and CPP dose.

animals. Additionally, reducing the number of stimuli presented on each trial simplified the procedure by reducing experimenter demands and session length while maintaining the variety of measures that can be obtained from the OST. Such task designs will be useful for investigating the time course of drug effects on memory, developmental changes in animal models of pathology, and localization of brain structures that process olfactory working memory. For example, Kesner et al. (2010) reported impaired accuracy in ventral CA1-lesioned rats for odors presented in close temporal proximity, indicating that the delay between an odor's initial and subsequent presentation influences memory for that odor. Our 24 odor two-comparison task will facilitate an extension of this approach with systematic assessments of memory for temporal delays interacting with the number of presentations and brain mechanisms of plasticity.

Among the challenges involved with developing translational models of psychopathology is the difficulty of comparing cognitive function across species (Gilmour et al. 2012). An advantage of the OST is that it has been adapted for rats (Dudchenko et al. 2000), mice (Young et al. 2007b), and humans (Levy et al. 2003). The consistency of findings with regard to the role of the NMDA-r in odor span performance indicates that the OST may be particularly useful in evaluating models of pathology associated with NMDA-r dysfunction. For example, expression and function of the NMDA-r has been linked to cognitive markers of schizophrenia (Linderholm et al. 2010; Zhou et al. 2010; Barnes et al. 2012; Gilmour et al. 2012). Considering the pharmacological effects observed here, the OST may serve as an assessment for investigating the interactive effects of cholinergic and glutamatergic signaling in animal models of schizophrenia (Dudchenko et al. 2012). The OST also shows potential utility in the assessment of Alzheimer's disease and attention deficit, as it can characterize long-term and working memory, as well as assess performance across memory load (Lehohla et al. 2004; Mota et al. 2013). Therefore, the translational merits of the OST indicate it can be a useful measure for assessment of cognitive deficits across species and neurological disorders.

In summary, we have reported that performance of 24 odor two-comparison dual-component OST is capacity dependent and is selectively impaired by the competitive NMDA-r antagonist, CPP. Our study confirms the involvement of the NMDA receptor in the OST and provides a foundation for the further assessment of the role of nicotinic receptors in the reversal of NMDA-r antagonist-induced impairments.

\section{Materials and Methods}

\section{Subjects}

Eight adult male Holtzman Sprague-Dawley rats (250-275 g) received from Charles River Laboratories were given $1 \mathrm{wk}$ to habituate to laboratory housing before training began. Rats were housed in pairs in a temperature and humidity controlled vivarium under a 12-h light-dark cycle and were given ad libitum access to water. Food access was restricted to maintain rats at $\sim 85 \%$ of free feeding weight to facilitate appetitive reinforcement. All procedures were approved by the Institutional Animal Care and Use Committee at the University of South Florida and conducted in accordance with principles of laboratory animal care and the National Institute of Health Guide for the Care and Use of Laboratory Animals.

\section{Apparatus}

Rats were tested in an open-field apparatus constructed from a circular table $(355 \mathrm{~cm}$ tall, $76 \mathrm{~cm}$ in diameter) with a Formica surface. The apparatus was encircled by a strip of sheet metal baffling which served as a wall around the open field $(32 \mathrm{~cm}$ high). Twelve holes $(5.5 \mathrm{~cm}$ in diameter) were drilled in the surface of the table to facilitate the presentation of stimuli. Stimulus presentation ports were evenly positioned along the edge of the table, 8 $\mathrm{cm}$ from the sheet metal (see Fig. 1). During sessions, an uncapped 2.0-oz plastic serving cup occupied any port not being used to present a stimulus. All sessions were recorded using a webcam centrally positioned $114 \mathrm{~cm}$ above the table. The apparatus was illuminated evenly using a $100 \mathrm{~W}$ bulb located above the table, unobstructed by the webcam.

\section{Stimuli}

The presentation of olfactory stimuli was accomplished by scenting plastic lids that corresponded to the 2.0-oz plastic service cups. Airtight plastic containers that held a single household spice below a platform were used to scent the plastic lids. Odorants included the following spices: allspice, anise, basil, beet, celery, chamomile, clove, coriander, cumin, fennel, fenugreek, garlic, ginger, horseradish, lavender, lemon balm, mustard, nutmeg, onion, orange, oregano, paprika, parsley, peppermint, rosemary, sage, thyme, and turmeric. Each lid was stored in an odorant container on top of the platform for at least $48 \mathrm{~h}$ before being used in a training or testing session. When presented as a stimulus, scented lids were placed on top of, but not snapped to, a matching 2.0-oz plastic cup that sat in one of the 12 stimulus presentation ports

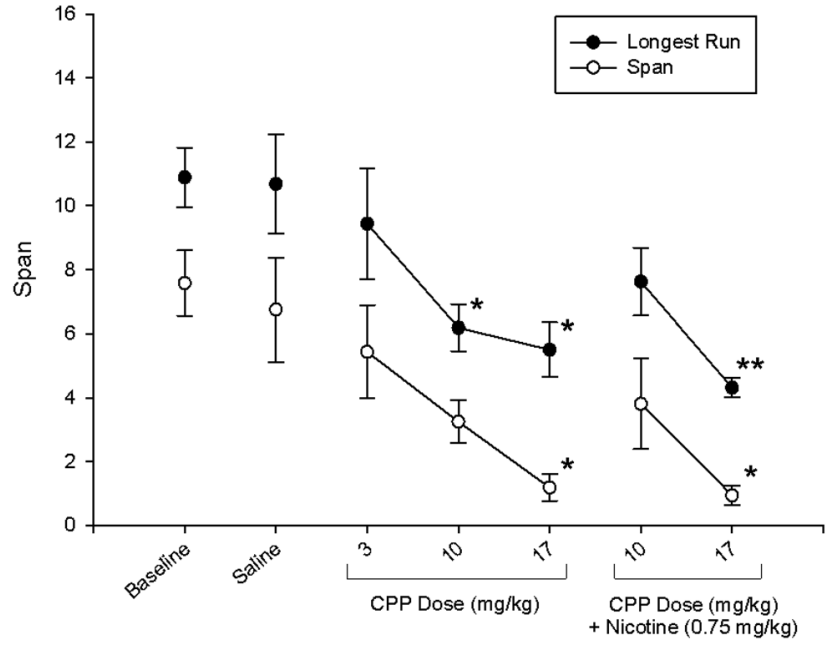

Figure 6. Average span and average longest run \pm SEM across dose conditions. Asterisks indicate a significant difference from saline $\left(\left(^{*}\right) P<\right.$ 0.05; (**) $P<0.01)$. 
indicated in Figure 1. To ensure that task performance was not affected by scent marking, lids were replaced after every trial with a fresh lid of the same scent such that each lid was only used once per session. To prevent the cup from being displaced from the port during stimulus responses, stimulus cups were weighted by filling two-thirds of the cup with white, fine grained sand.

\section{Procedure}

The sequence of training events is illustrated in Figure 1B. An average of 25 sessions ( 1 session/day) were required for the rats to meet the criteria for all four training procedures and advance to the drug phase. The total duration of preliminary training followed by drug testing was an average of $76.1 \mathrm{~d} /$ rat over $5.7 \mathrm{mo}$.

\section{Pretraining}

After a 1-wk handling habituation period (30 min/day), each rat was habituated to the arena by allowing it to freely explore the apparatus and consume $45 \mathrm{mg}$ sucrose pellets from uncapped stimulus cups during a 30-min session for several days. When a rat consistently consumed pellets in the arena, it was transitioned to a shaping procedure for removing stimulus lids. This consisted of placing the rat in the arena with a single stimulus cup, baited with a sucrose pellet, and an unscented lid placed adjacent to it. After the rat discovered and consumed the pellet, it was removed from the apparatus and the stimulus cup was moved to a randomized port location. The lid was then placed on top of the baited cup so that it partially covered its opening and, on each subsequent trial, was moved to cover more and more of the cup opening. Rats were trained in this phase until they successfully removed lids from fully obscured stimulus cups on every trial of a 30-min session ( 2 min-max per trial). In all subsequent training and testing sessions, odors were presented via scented lids which fully obscured, but were not snapped onto, stimulus cups.

\section{Span training}

In this phase, rats began incrementing olfactory nonmatch to sample (NMTS) training. On the first trial of the session, a single scented lid was presented on top of a baited stimulus cup in a randomized port location while empty cups without lids occupied the other 11 ports. The trial began when the rat was placed in the arena facing location 6 (see Fig. 1), and was terminated when the rat displaced the lid of the stimulus cup using any part of the upper body (nose, arms, etc.). Following a response, the rat was moved to a holding cage for an intertrial interval of $\sim 30 \mathrm{sec}$. During this time, stimuli were rearranged such that the odor presented in Trial 1 and a novel odor were presented, with both cup locations randomized. Only the stimulus cup featuring the novel odor was baited with a sucrose pellet.

If the rat responded to the novel stimulus, it was allowed to retrieve and consume the sucrose pellet before being returned to the holding cage. Following a correct response, three odors were presented in the apparatus: The two odors used in the preceding trials (unbaited) and a novel odor (baited), $\mathrm{S}-$ and $\mathrm{S}+$, respectively. After each correct response, the subsequent trial included a novel odor $(\mathrm{S}+)$ presented along with all of the odors used on the previous trial $(\mathrm{S}-)$.

If the rat responded to a comparison $(\mathrm{S}-)$ stimulus, the trial was ended and scored as an error. After each error, span was computed by tallying the number of consecutive correct responses before the error and subtracting one. One correct response must be subtracted from the tallied span as the rat cannot produce an error on the first trial of a span (apart from failing to respond) because only a single stimulus, an $\mathrm{S}+$, is presented. After an error, sessions were continued by beginning the incrementing NMTS procedure again using odors that did not appear in the previous trials. This procedure was repeated until 24 trials or $30 \mathrm{~min}$ had elapsed. As the apparatus only contained 12 stimulus presentation ports, previously presented stimuli were randomly chosen to be omitted from the comparison array when spans exceeded 11 . This procedure was also used in the next training phase (12-comparison span). Rats were continued on span training until a span of five or greater was produced during three consecutive sessions. An average of $8.9(\mathrm{SEM}=1.0)$ sessions were required to reach criterion and advance to the next stage of training.

\section{Twelve-comparison span}

The procedure for this phase of training was identical to span training with the exception that stimuli continued to increment for 24 trials regardless of performance to acclimate rats to the continuous procedure used in future trials. To ensure that rats sampled the novel odor on every trial, rats were allowed to respond to the $\mathrm{S}+$ after an error was made (a response to an $\mathrm{S}-$ ) and the trial was not terminated until the rat responded to the $S+$. Both span and percent correct performance were recorded during this phase. Rats advanced to the next phase of training when a span of 5 or greater was produced during three consecutive sessions. An average of $4.3(\mathrm{SEM}=0.5)$ sessions were required to reach criterion and advance to the next stage of training.

\section{Reference memory}

After completing the 12-comparison span training, rats were trained on a reference memory procedure using two odors chosen from a set of odors not used in the OST (lavender, garlic, peppermint, and orange peel). For each rat one odor, selected as the $\mathrm{S}+$, was always presented with a baited stimulus cup, while the other, assigned to be the $S-$, was never baited. In each RM training trial both odors were presented in random locations within the arena. Rats were free to respond to either stimuli, but the trial was terminated after the first response, regardless of performance. Rats received 20 trials of RM training per session until they qualified for the dual-component training with accuracy equal to or better than $75 \%$ on three consecutive RM training sessions. RM trials were learned for future integration into the dual-component, twocomparison span as a within session assessment of long-term memory ability as well as motivational and sensorimotor impairments that may result from drug administration. An average of 4.4 $(\mathrm{SEM}=0.5)$ sessions were required for subjects to reach criterion and advance to the next stage of training.

\section{Dual component, 24 odor two-comparison span}

Once a rat met criterion for the RM training, it was advanced to the dual-component, 24 odor two-comparison span training, which consisted of RM trials interspersed within a 24 trial, 24 odor two-comparison NMTS OST session. Training continued as in the 12-comparison span phase with the exception that no more than two odor stimuli were presented in the arena on any trial such that one novel odor and one comparison odor randomly chosen from the odors used in preceding trials were presented. By presenting only two stimuli per trial, chance performance $(50 \%)$ was equated across all trials of the OST. In earlier versions of the task, chance performance decreases as trials progress given that the number of comparison stimuli presented increases on each trial. As such, chance performance is confounded with the number of stimuli to remember. Equating chance performance across trials allows for a meaningful analysis of the relationship between performance and memory load.

In addition to the 24 odor two-comparison OST, sessions included six RM trials scattered throughout the session after every fourth odor span trial. RM stimuli remained constant for each rat across all training and testing sessions. As accurate performance on the RM trials relied on information acquired from distant prior training, they served as an assessment of long-term memory capability. Additionally, accurate RM performance demonstrated that the rat was able to navigate the apparatus, discriminate between odors, produce an appropriate response, and was sufficiently motivated to do so. Thus, RM trials served as a within session control for sensorimotor or motivational disruption, and gross cognitive impairment.

Each session consisted of 30 trials: 24 trials of two-comparison OST and six RM trials. During this phase and 
subsequent testing rats were given a maximum of 2 min each trial to produce a response. Failure to provide a response resulted in termination of the trial and an "omission" was noted by the experimenter. Dual-component training was continued until asymptotic criteria was met which required that the average percent correct performance over the past three sessions did not differ from the average percent correct performance of the preceding three sessions by $>15 \%$ of the average of all six sessions. An average of $7.5(\mathrm{SEM}=0.5)$ sessions were required for subjects to reach criterion and advance to the drug phase.

\section{Drug phase}

After reaching asymptotic level in the dual-component, 24 odor two-comparison OST, each rat received between 1 and 3 practice injections with saline before beginning drug trials to acclimate to the injection procedure. Rats were tested on the dualcomponent, two-comparison OST procedure on Monday through Friday with drug administrations on Tuesdays and Fridays. Doses of CPP were prepared by dissolving CPP in $0.9 \%$ saline to produce dose concentrations of 3,10 , and $17 \mathrm{mg} / \mathrm{kg}$ (expressed as total salt). Each dose of CPP was delivered intraperitoneously $(1 \mathrm{~mL} /$ $\mathrm{kg}) 30 \mathrm{~min}$ prior to testing. During each drug trial, the experimenter responsible for testing was blind to the dose of CPP delivered. Each rat received two administrations of each dose and saline in a random order with the exception that each dose was delivered once before a second cycle began. After receiving two sessions of each dose of CPP, rats were given combinations of CPP (10 and $17 \mathrm{mg} / \mathrm{kg}$ ) and nicotine $(0.75 \mathrm{mg} / \mathrm{kg})$. Nicotine was prepared for injections by dissolving $(-)$-nicotine hydrogen tartrate salt in $0.9 \%$ saline and were delivered via intraperitoneal injection (1 $\mathrm{mL} / \mathrm{kg}$ ) $15 \mathrm{~min}$ after the rat received CPP, which corresponded to 15 min before testing. Each rat received two administrations of both combinations of CPP $(10,17 \mathrm{mg} / \mathrm{kg})$ and nicotine $(0.75$ $\mathrm{mg} / \mathrm{kg}$ ) for a total of four interaction sessions.

\section{Data analysis}

Four primary outcome measures were derived from each session: span, longest run, percent correct accuracy for OST and RM trials, and latency to choice. On each choice trial, the experimenter also recorded whether the subject approached one or both odors; an approach was scored when the subject's nose came within 25 $\mathrm{mm}$ of the lid. Span was defined as the number of correct responses from the beginning of the session until the first error, minus 1 . The longest run was defined as the longest string of consecutive correct responses during the session. Percent correct accuracy was computed both for OST trials and for RM trials. However, the first trial of the OST was omitted from all analyses of accuracy as no choice is presented on this trial. Within subjects, measures were averaged across the two determinations at each drug condition and across baseline sessions (sessions after stability where no injections were administered). Accuracy was also averaged across sessions at each trial number so that the relationship between memory load and accuracy could be assessed. Accuracy across the session was also compared across dose. Because subjects completed fewer sessions under the dose conditions (as compared with baseline) performance was averaged across blocks of four trials to produce a more reliable estimate of performance. On occasion, rats failed to produce a response within the 2-min time window. Omissions were recorded and are also reported by condition. Trials resulting in an omission were not included when averaging latency. Dose effects were evaluated with repeated measures analysis of variance (ANOVA). For instances in which a violation of sphericity was observed, a Greenhouse-Geisser correction was used as a more stringent test of significance (Gamst et al. 2008, pp. 269-270).

\section{Competing interest statement}

None declared.

\section{Acknowledgments}

This research was supported by Career Scientist and Merit Review Awards from the Biomedical Laboratory Research \& Development Service of the Department of Veterans Affairs to D.M.D. The opinions expressed in this paper are those of the authors and not of the Department of Veterans Affairs or the U.S. government.

\section{References}

April LB, Bruce K, Galizio M. 2013. The magic number 70 (plus or minus 20): variables determining performance in the Rodent Odor Span Task. Learn Motiv 44: 143-158.

Baddeley A. 2003. Working memory: looking back and looking forward. Nat Rev Neurosci 4: 829-839.

Barnes SA, Young JW, Neill JC. 2012. Rats tested after a washout period from sub-chronic PCP administration exhibited impaired performance in the 5-Choice Continuous Performance Test (5C-CPT) when the attentional load was increased. Neuropharmacology 62: 1432-1441.

Brun VH, Ytterbo K, Morris RG, Moser MB, Moser EI. 2001. Retrograde amnesia for spatial memory induced by NMDA receptor-mediated long-term potentiation. J Neurosci 21: 356-362.

Cui Y, Jin J, Zhang X, Xu H, Yang L, Du D, Zeng Q, Tsien JZ, Yu H, Cao X. 2011. Forebrain NR2B overexpression facilitating the prefrontal cortex long-term potentiation and enhancing working memory function in mice. PLoS One 6: e20312.

Davies DA, Greba Q, Howland JG. 2013a. GluN2B-containing NMDA receptors and AMPA receptors in medial prefrontal cortex are necessary for odor span in rats. Front Behav Neurosci 7: 183.

Davies DA, Molder JJ, Greba Q, Howland JG. 2013b. Inactivation of medial prefrontal cortex or acute stress impairs odor span in rats. Learn Mem 20: $665-669$.

Dudchenko PA, Wood ER, Eichenbaum H. 2000. Neurotoxic hippocampal lesions have no effect on odor span and little effect on odor recognition memory but produce significant impairments on spatial span, recognition, and alternation. J Neurosci 20: 2964-2977.

Dudchenko PA, Talpos J, Young J, Baxter MG. 2012. Animal models of working memory: a review of tasks that might be used in screening drug treatments for the memory impairments found in schizophrenia. Neurosci Biobehav Rev 37: 2111-2124.

Feldman LA, Shapiro ML, Nalbantoglu J. 2010. A novel, rapidly acquired and persistent spatial memory task that induces immediate early gene expression. Behav Brain Funct 6: 35.

Fortin NJ, Agster KL, Eichenbaum HB. 2002. Critical role of the hippocampus in memory for sequences of events. Nat Neurosci 5: $458-462$.

Galizio M, Deal M, Hawkey A, April B. 2013. Working memory in the odor span task: effects of chlordiazepoxide, dizocilpine (MK801), morphine, and scopolamine. Psychopharmacology (Berl) 225: 397-406.

Gamst G, Meyers LS, Guarino AJ. 2008. Analysis of variance designs: a conceptual and computational approach with SPSS and SASS. Cambridge University Press, New York.

Gilmour G, Dix S, Fellini L, Gastambide F, Plath N, Steckler T, Talpos J, Tricklebank M. 2012. NMDA receptors, cognition and schizophrenia-testing the validity of the NMDA receptor hypofunction hypothesis. Neuropharmacology 62: 1401-1412.

Hawkey A, April LB, Galizio M. 2014. Effects of MDMA on olfactory memory and reversal learning in rats. Neurobiol Learn Mem 114: 209-216.

Heishman SJ, Kleykamp BA, Singleton EG. 2010. Meta-analysis of the acute effects of nicotine and smoking on human performance. Psychopharmacology (Berl) 210: 453-469.

Kesner RP, Gilbert PE, Barua LA. 2002. The role of the hippocampus in memory for the temporal order of a sequence of odors. Behav Neurosci 116: $286-290$.

Kesner RP, Hunsaker MR, Ziegler W. 2010. The role of the dorsal CA1 and ventral CA1 in memory for the temporal order of a sequence of odors. Neurobiol Learn Mem 93: 111-116.

Kesner RP, Hunsaker MR, Ziegler W. 2011. The role of the dorsal and ventral hippocampus in olfactory working memory. Neurobiol Learn Mem 96: $361-366$.

Larkin AE, Fahey B, Gobbo O, Callaghan CK, Cahill E, O'Mara SM, Kelly AM. 2008. Blockade of NMDA receptors pre-training, but not post-training, impairs object displacement learning in the rat. Brain Res 1199: $126-132$.

Lehohla M, Kellaway L, Russell VA. 2004. NMDA receptor function in the prefrontal cortex of a rat model for attention-deficit hyperactivity disorder. Metab Brain Dis 19: 35-42.

Levy DA, Manns JR, Hopkins RO, Gold JJ, Broadbent NJ, Squire LR. 2003. Impaired visual and odor recognition memory span in patients with hippocampal lesions. Learn Mem 10: 531-536. 
Linderholm K, Powell S, Olsson E, Holtze M, Snodgrass R, Erhardt S. 2010. Role of the NMDA-receptor in prepulse inhibition in the rat. Int $J$ Tryptophan Res 3: 1-12.

MacQueen DA, Bullard L, Galizio M. 2011. Effects of dizocilpine (MK801) on olfactory span in rats. Neurobiol Learn Mem 95: 57-63.

Martin SJ, Clark RE. 2007. The rodent hippocampus and spatial memory: from synapses to systems. Cell Mol Life Sci 64(4): 401-431.

Mota SI, Ferreira IL, Rego AC. 2013. Dysfunctional synapse in Alzheimer's disease - a focus on NMDA receptors. Neuropharmacology.

Niimi K, Takahashi E, Itakura C. 2008. Improved short-term memory and increased expression of NR2B observed in senescence-accelerated mouse (SAM) P6. Exp Gerontol 43: 847-852.

Olton DS, Feustle WA. 1981. Hippocampal function required for nonspatial working memory. Exp Brain Res 41: 380-389.

Rushforth SL, Allison C, Wonnacott S, Shoaib M. 2010. Subtype-selective nicotinic agonists enhance olfactory working memory in normal rats: a novel use of the odour span task. Neurosci Lett 471: $114-118$.

Rushforth SL, Steckler T, Shoaib M. 2011. Nicotine improves working memory span capacity in rats following sub-chronic ketamine exposure. Neuropsychopharmacology 36: 2774-2781.
Velazquez-Zamora DA, Gonzalez-Ramirez MM, Beas-Zarate C, Gonzalez-Burgos I. 2011. Egocentric working memory impairment and dendritic spine plastic changes in prefrontal neurons after NMDA receptor blockade in rats. Brain Res 1402: 101-108.

Young JW, Crawford N, Kelly JS, Kerr LE, Marston HM, Spratt C, Finlayson K, Sharkey J. 2007a. Impaired attention is central to the cognitive deficits observed in $\alpha 7$ deficient mice. Eur Neuropsychopharmacol 17: 145-155.

Young JW, Kerr LE, Kelly JS, Marston HM, Spratt C, Finlayson K, Sharkey J. 2007b. The odour span task: a novel paradigm for assessing working memory in mice. Neuropharmacology 52: 634-645.

Zhou X, Nie Z, Roberts A, Zhang D, Sebat J, Malhotra D, Kelsoe JR, Geyer MA. 2010. Reduced NMDAR1 expression in the Sp4 hypomorphic mouse may contribute to endophenotypes of human psychiatric disorders. Hum Mol Genet 19: 3797-3805.

Received December 1, 2015; accepted in revised form March 13, 2016. 


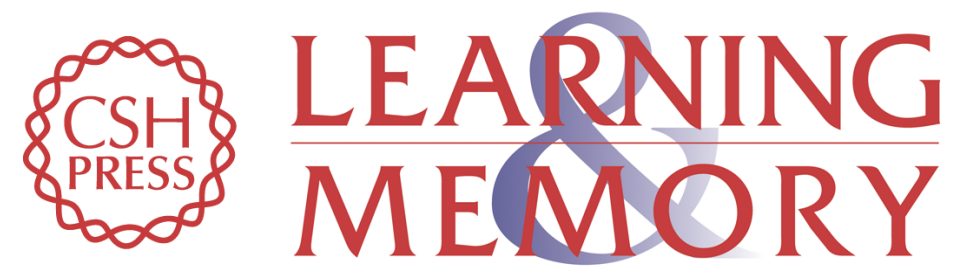

\section{Influence of pharmacological manipulations of NMDA and cholinergic receptors on working versus reference memory in a dual component odor span task}

David A. MacQueen, Savannah R. Dalrymple, David J. Drobes, et al.

Learn. Mem. 2016, 23:

Access the most recent version at doi:10.1101//m.041251.115

References This article cites 32 articles, 4 of which can be accessed free at: http://learnmem.cshlp.org/content/23/6/270.full.html\#ref-list-1

Creative This article is distributed exclusively by Cold Spring Harbor Laboratory Press for the Commons first 12 months after the full-issue publication date (see

License http://learnmem.cshlp.org/site/misc/terms.xhtml). After 12 months, it is available under a Creative Commons License (Attribution-NonCommercial 4.0 International), as described at http://creativecommons.org/licenses/by-nc/4.0/.

Email Alerting Receive free email alerts when new articles cite this article - sign up in the box at the Service top right corner of the article or click here. 